\title{
SKELETONS OF MONOMIAL IDEALS
}

\author{
JÜRGEN HERZOG, ALI SOLEYMAN JAHAN AND XINXIAN ZHENG
}

\begin{abstract}
In analogy to the skeletons of a simplicial complex and their Stanley-Reisner ideals we introduce the skeletons of an arbitrary monomial ideal $I \subset S=K\left[x_{1}, \ldots, x_{n}\right]$. This allows us to compute the depth of $S / I$ in terms of its skeleton ideals. We apply these techniques to show that Stanley's conjecture on Stanley decompositions of $S / I$ holds provided it holds whenever $S / I$ is Cohen-Macaulay. We also discuss a conjecture of Soleyman-Jahan and show that it suffices to prove his conjecture for monomial ideals with linear resolution.
\end{abstract}

\section{INTRODUCTION}

Let $\Delta$ be a simplicial complex of dimension $d-1$ on the vertex set $\{1, \ldots, n\}, K$ a field and $K[\Delta]$ the Stanley-Reisner ring of $\Delta$. The depth of $K[\Delta]$ can be expressed in terms of the skeletons of $\Delta$, as has been shown by D. Smith [6, Theorem 3.7] for pure simplicial complexes, and by Hibi [5, Corollary 2.6] in general. The $j$ th skeleton of $\Delta$ is the simplicial subcomplex $\Delta^{(j)}=\{F \in \Delta:|F| \leq j\}$ of $\Delta$. The result is that $\operatorname{depth} K[\Delta]=\max \left\{j: \Delta^{(j)}\right.$ is Cohen-Macaulay $\}$.

The purpose of this paper is to generalize this result as follows: first note that we have the following chain of Stanley-Reisner ideals $I_{\Delta}=I_{\Delta^{d}} \subset I_{\Delta^{d-1}} \subset \cdots \subset I_{\Delta^{0}} \subset S$ with $\operatorname{dim} S / I_{\Delta^{(j)}}=j$ for all $j$. Now for an arbitrary monomial ideal $I \subset S$ we want to define in a natural way a similar chain of monomial ideals $I=I_{d} \subset I_{d-1} \subset \cdots \subset I_{0} \subset S$ with $\operatorname{dim} S / I_{j}=j$ for all $j$, and of course this chain should satisfy the condition that $\operatorname{depth} S / I=\max \left\{j: S / I_{j}\right.$ is Cohen-Macaulay $\}$. We show in Section 1 that such a natural chain of monomial ideals with these properties indeed exists. The ideal $I_{j}$ is called the $j$ th skeleton ideal of $I$.

For the construction of the skeleton ideals of $I$ we consider the so-called characteristic poset $P_{S / I}^{g}$ introduced in [4]. Here $g \in \mathbb{N}^{n}$ is an integer vector such that $g \geq a$ for all $a$ for which $x^{a}$ belongs to the minimal set of monomial generators of $I$, and $P_{S / I}^{g}$ is the (finite) poset of all $b \in \mathbb{N}^{n}$ such that $b \leq g$ and $x^{b} \notin I$. Here the partial order on $\mathbb{N}^{n}$ is defined as follows: $a \leq b$ if and only if $a(i) \leq b(i)$ for $i=1, \ldots, n$. In case of a Stanley-Reisner ideal $I_{\Delta}$ and $g=(1,1, \ldots, 1)$ this poset is just the face poset of $\Delta$. For

1991 Mathematics Subject Classification. 13C13, 13C14, 05E99, 16 W70.

Key words and phrases. Monomial ideals, depth, skeleton, Cohen-Macaulay, Stanley decompositions.

The third author is grateful for the financial support by DFG (Deutsche Forschungsgemeinschaft) during the preparation of this work. 
each $b \in \mathbb{N}^{n}$, let $\rho(b)=|\{j: b(j)=g(j)\}|$. It has been shown in [4, Corollary 2.6] that $\operatorname{dim} S / I=\max \left\{\rho(b): \quad b \in P_{S / I}^{g}\right\}$. We use this integer function $\rho$ to define the skeleton ideals of $I$, and let $I_{j}$ be the monomial ideal generated by $I$ and all $x^{b}$ with $\rho(b)>j$. It is the easy to see that $\operatorname{dim} S / I_{j}=j$ for all $j$. The crucial result however is that for all $j, I_{j-1} / I_{j}$ is Cohen-Macaulay module of dimension $j$, see Theorem 1.2. From this result we easily deduce in Corollary [1.5] a generalization of the result of Hibi, namely that $\operatorname{depth} S / I=\max \left\{j: S / I_{j}\right.$ is Cohen-Macaulay $\}$.

In Section 2 we apply the results and techniques introduced in Section 1 to deduce some results on Stanley decomposition. Let $M$ be a finitely generated $\mathbb{Z}^{n}$-graded $S$-module, $m \in M$ be a homogeneous element and $Z \subset X=\left\{x_{1}, \ldots, x_{n}\right\}$. We denote by $m K[Z]$ the $K$-subspace of $M$ generated by all homogeneous elements of the form $m u$, where $u$ is a monomial in $K[Z]$. The $K$-subspace $m K[Z]$ is called a Stanley space of dimension $|Z|$ if $m K[Z]$ is a free $K[Z]$-module.

A decomposition $\mathcal{D}$ of $M$ as a finite direct sum of Stanley spaces is called a Stanley decomposition of $M$. The minimal dimension of a Stanley space in the decomposition $\mathcal{D}$ is called the Stanley depth of $\mathcal{D}$, denoted by $\operatorname{sdepth} \mathcal{D}$. We set

$$
\text { sdepth } M=\max \{\operatorname{sdepth} \mathcal{D}: \mathcal{D} \text { is a Stanley decomposition of } M\} \text {, }
$$

and call this number the Stanley depth of $M$. A famous conjecture of Stanley asserts that sdepth $M \geq \operatorname{depth} M$.

As one of the main results of Section 2 we show in Corollary 2.2 that for each monomial ideal $I$ Stanley's conjecture holds for $S / I$ if it holds whenever $S / I$ is Cohen-Macaulay. We also discuss a conjecture of Soleyman-Jahan. His conjecture asserts that we can always find a Stanley decomposition $M=\bigoplus_{j=1}^{r} m_{j} K\left[Z_{j}\right]$ of $M$ with $\left|\operatorname{deg} m_{j}\right| \leq \operatorname{reg}(M)$ for all $j$. Here $|a|=\sum_{i=1}^{n} a(i)$ for $a \in \mathbb{Z}^{n}$. We show in the case that $M=I$ is a monomial ideal, it suffices to prove this conjecture when $I$ has a linear resolution.

\section{Characteristic posets and skeletons}

Let $K$ be a field, $S=K\left[x_{1}, \ldots, x_{n}\right]$ the polynomial ring in $n$ variables and $I \subset S$ a monomial ideal. We denote by $G(I)$ the unique minimal set of monomial generators of $I$. Let $G(I)=\left\{u_{1}, \ldots, u_{m}\right\}$ with $u_{i}=x^{a_{i}}$ and $a_{i} \in \mathbb{N}^{n}$. Here, for any $c \in \mathbb{N}^{n}$ we denote as usual by $x^{c}$ the monomial $x_{1}^{c(1)} x_{2}^{c(2)} \cdots x_{n}^{c(n)}$.

Observe that $\mathbb{N}^{n}$ with the natural partial order introduced in the introduction is a distributive lattice with meet $a \wedge b$ and join $a \vee b$ defined as follows: $(a \wedge b)(i)=\min \{a(i), b(i)\}$ and $(a \vee b)(i)=\max \{a(i), b(i)\}$. We also denote by $\varepsilon_{j}$ the $j$ th canonical unit vector in $\mathbb{Z}^{n}$.

Let $J \subset S$ be another monomial ideal with $I \subset J$, minimally generated by $x^{b_{1}}, \ldots, x^{b_{s}}$. We choose $g \in \mathbb{N}^{n}$ such that $a_{i} \leq g$ and $b_{j} \leq g$ for all $i$ and $j$, and let $P_{J / I}^{g}$ be the set of all $b \in \mathbb{N}^{n}$ with $b \leq g, b \geq b_{j}$ for some $j$, and $b \nsupseteq a_{i}$ for all $i$. The set $P_{J / I}^{g}$ viewed as a 
subposet of $\mathbb{N}^{n}$ is a finite poset, and is called the characteristic poset of $J / I$ with respect to $g$, see [4].

For any $b \in \mathbb{N}^{n}$ we define subsets $Y_{b}=\left\{x_{j}: b(j) \neq g(j)\right\}$ and $Z_{b}=\left\{x_{j}: b(j)=g(j)\right\}$ of $X=\left\{x_{1}, \ldots, x_{n}\right\}$ and set $\rho(b)=|\{j: b(j)=g(j)\}|=\left|Z_{b}\right|$. Let $d=\operatorname{dim} J / I$ be the Krull dimension of $J / I$. It is shown in [4, Corollary 2.6] that

$$
d=\max \left\{\rho(b): b \in P_{J / I}^{g}\right\} .
$$

As a consequence of (11) we obtain

Lemma 1.1. Let $d=\operatorname{dim} J / I$. Then $\rho(b) \leq d$ for all $b \in \mathbb{N}^{n}$ with $x^{b} \in J \backslash I$.

Proof. Let $g^{\prime}=g \vee b$. Then $b \in P_{J / I}^{g^{\prime}}$, and hence $\left|\left\{j: b(j)=g^{\prime}(j)\right\}\right| \leq d$, by (11). Since $\rho(b)=|\{j: \quad b(j)=g(j)\}| \leq|\{j: \quad b(j) \geq g(j)\}|=\left|\left\{j: \quad b(j)=g^{\prime}(j)\right\}\right|$, the assertion follows.

Formula (10) leads us to consider for each $j \leq d$, the monomial ideal $I_{j}$ generated by $I$ together with all monomials $x^{b}$ such that $\rho(b)>j$. We then obtain a chain of monomial ideals

$$
I=I_{d} \subset I_{d-1} \subset \cdots \subset I_{0} \subset S .
$$

Of course this chain of ideals depends not only on $I$, but also on the choice of $g$.

Consider the special case, where $I=I_{\Delta}$ is the Stanley-Reisner ideal of a simplicial complex $\Delta$ on the vertex set $\{1, \ldots, n\}$. Then for $g=(1, \ldots, 1)$ we have $I_{j}=I_{\Delta^{(j)}}$. This observation justifies to call $I_{j}$ the $j$ th skeleton ideal of $I$ (with respect to $g$ ).

The following result is crucial for this note.

Theorem 1.2. For each $0 \leq j \leq d$, the factor module $I_{j-1} / I_{j}$ is a direct sum of cyclic Cohen-Macaulay modules of dimension $j$. In particular, $I_{j-1} / I_{j}$ is a $j$-dimensional Cohen-Macaulay module.

Proof. Replacing $I$ by $I_{j}$ it suffices to consider the case $j=d$. Let

$$
J=\left(I,\left\{x^{b}: b \in A\right\}\right), \quad \text { where } \quad A=\left\{b \in P_{S / I}^{g}: \rho(b)=d\right\},
$$

then $I_{d-1} / I_{d}=J / I$.

Let $\left\{Z_{1}, \ldots, Z_{r}\right\}$ be the collection of those subsets of $X$ with the property that for each $i=1, \ldots, r$ there exists $b \in A$ such that $Z_{i}=Z_{b}$. Let $A_{i}=\left\{b \in A: Z_{b}=Z_{i}\right\}$, and let $b, b^{\prime} \in A_{i}$. Then $b \wedge b^{\prime} \in A_{i}$. Thus the meet of all the elements in $A_{i}$ is the unique smallest element in $A_{i}$. We denote this element by $b_{i}$. Then $Z_{i}=Z_{b_{i}}$. Obviously the elements $f_{i}=x^{b_{i}}+I, i=1, \ldots, r$ generate $J / I$. We claim that

$$
J / I=\bigoplus_{i=1}^{r} S f_{i} .
$$


The cyclic module $S f_{i}$ is $\mathbb{Z}^{n}$-graded with a $K$-basis $x^{a}+I$ with $a \geq b_{i}$ and $x^{a} \notin I$. Given $c \in \mathbb{N}^{n}$ with $c \geq b_{i}$ and $c \geq b_{j}$ for some $1 \leq i<j \leq r$, then $\rho(c)>d$, and so $x^{c} \in I$, by Lemma 1.1. This shows that the sum of the cyclic modules $S f_{i}$ is indeed direct.

Next we notice that if $x^{c}=x^{c_{1}} x^{c_{2}}$ with $x^{c_{1}} \in K\left[Z_{b_{i}}\right]$ and $x^{c_{2}} \in K\left[Y_{b_{i}}\right]$ belongs to $\operatorname{Ann}\left(S f_{i}\right)$, then $x^{c_{2}} \in \operatorname{Ann}\left(S f_{i}\right)$. Indeed, $x^{c}=x^{c_{1}} x^{c_{2}} \in \operatorname{Ann}\left(S f_{i}\right)$ if and only if $a_{j} \leq$ $b_{i}+c_{1}+c_{2}$ for some $j$. Since $c_{1}(k)=0$ for all $k$ with $x_{k} \in Y_{b_{i}}$, it follows that $a_{j}(k) \leq$ $\left(b_{i}+c_{2}\right)(k)$ for all $k \in Y_{b_{i}}$, while for $k$ with $x_{k} \in Z_{b_{i}}$ we have $a_{j}(k) \leq g(k)=b_{i}(k)=$ $\left(b_{i}+c_{2}\right)(k)$. Hence $a_{j} \leq b_{i}+c_{2}$, which implies that $x^{c_{2}} \in \operatorname{Ann}\left(S f_{i}\right)$.

It follows that $\operatorname{Ann}\left(S f_{i}\right)$ is generated by monomials in $K\left[Y_{b_{i}}\right]$. In other words, there exists a monomial ideal $M_{i} \subset K\left[Y_{b_{i}}\right]$ such that $\operatorname{Ann}\left(S f_{i}\right)=M_{i} S$.

For each $k$ with $x_{k} \in Y_{b_{i}}$ we have $b_{i}(k)<g(k)$ and $\rho\left(b_{i}+\left(g(k)-b_{i}(k)\right) \varepsilon_{k}\right)=d+1$. Therefore Lemma 1.1 implies that $x^{b_{i}} x_{k}^{g(k)-b_{i}(k)} \in I$. It follows that $x_{k}^{g(k)-b_{i}(k)} \in M_{i}$ for all $k$ with $x_{k} \in Y_{b_{i}}$. Hence we see that $\operatorname{dim} K\left[Y_{b_{i}}\right] / M_{i}=0$. This implies that $S f_{i}=S / M_{i} S$ is Cohen-Macaulay of dimension $d$.

Remark 1.3. It is also possible to define skeletons of $J / I$ in the same way as for $S / I$, and one obtains a chain of ideals $I=I_{d} \subset I_{d-1} \subset \cdots \subset I_{0} \subset J$. Some of the factor modules $I_{j-1} / I_{j}$ however may be 0 in this generality. But whenever $I_{j-1} / I_{j} \neq 0$ it follows again that $I_{j-1} / I_{j}$ is Cohen-Macaulay of dimension $j$, though not always a direct sum of cyclic modules.

Corollary 1.4. For $j=0 \ldots, d-1$ and $i=0 \ldots, j-1$, we have $\operatorname{depth}\left(I_{j} / I\right) \geq j+1$, and $H_{\mathfrak{m}}^{i}(S / I) \cong H_{\mathfrak{m}}^{i}\left(S / I_{j}\right)$.

Proof. We prove the assertion by induction on $d-j$. For $j=d-1$ the assertion follows from Theorem 1.2. Let $j<d-1$. Then the exact sequence

$$
0 \longrightarrow I_{j+1} / I \longrightarrow I_{j} / I \longrightarrow I_{j} / I_{j+1} \longrightarrow 0
$$

implies that

$$
\operatorname{depth}\left(I_{j} / I\right) \geq \min \left\{\operatorname{depth}\left(I_{j+1} / I\right), \operatorname{depth}\left(I_{j} / I_{j+1}\right)\right\}
$$

see [2, Proposition 1.2.9]. By Theorem [1.2, $\operatorname{depth}\left(I_{j} / I_{j+1}\right)=j+1$ and by induction hypothesis $\operatorname{depth}\left(I_{j+1} / I\right) \geq j+2$. Hence $\operatorname{depth}\left(I_{j} / I\right) \geq j+1$.

The short exact sequence

$$
0 \longrightarrow I_{j} / I \longrightarrow S / I \longrightarrow S / I_{j} \longrightarrow 0
$$

yields the long exact sequence

$$
\cdots \longrightarrow H_{\mathfrak{m}}^{i}\left(I_{j} / I\right) \longrightarrow H_{\mathfrak{m}}^{i}(S / I) \longrightarrow H_{\mathfrak{m}}^{i}\left(S / I_{j}\right) \longrightarrow H_{\mathfrak{m}}^{i+1}\left(I_{j} / I\right) \longrightarrow \cdots
$$

of local cohomology. By the first part of the statement we have $H_{\mathfrak{m}}^{k}\left(I_{j} / I\right)=0$ for $k \leq j$. This yields the desired isomorphisms.

As an application of Theorem 1.2 we obtain the following characterization of the depth of $S / I$ which generalizes a classical result of Hibi [5, Corollary 2.6]. 
Corollary 1.5. Let $I \subset S$ be a monomial ideal. Then

$$
\operatorname{depth} S / I=\max \left\{j: S / I_{j} \text { is Cohen-Macaulay }\right\} \text {, }
$$

and $S / I_{j}$ is Cohen-Macaulay for all $j \leq \operatorname{depth} S / I$.

Proof. Let $d=\operatorname{dim} S / I$ and $t=\operatorname{depth} S / I$. Since $I_{j}=\left(I_{d-1}\right)_{j}$ for $j \leq d-1$, both assertions follow by induction on $d$ once we can show the following:

(i) If $t<d$, then $\operatorname{depth} S / I_{d-1}=t$.

(ii) If $S / I$ is Cohen-Macaulay, then $S / I_{d-1}$ is Cohen-Macaulay.

Proof of (i): The exact sequence

$$
0 \longrightarrow I_{d-1} / I \longrightarrow S / I \longrightarrow S / I_{d-1} \longrightarrow 0
$$

implies that

$$
\operatorname{depth} S / I_{d-1} \geq \min \left\{\operatorname{depth}\left(I_{d-1} / I\right)-1, \operatorname{depth} S / I\right\},
$$

with equality if $t<d-1$, see [2, Proposition 1.2.9]. By Theorem 1.2, $\operatorname{depth}\left(I_{d-1} / I\right)-1=$ $d-1$. It follows that depth $S / I_{d-1}=t$, if $t<d-1$. On the other hand, if $t=d-1$, then $\operatorname{depth} S / I_{d-1} \geq d-1$. However, since $\operatorname{dim} S / I_{d-1}=d-1$, we again get $\operatorname{depth} S / I_{d-1}=$ $d-1=t$.

Proof of (ii): If $S / I$ is Cohen-Macaulay, then depth $S / I=d$. Hence Theorem 1.2 and inequality (2) imply that $\operatorname{depth} S / I_{d-1} \geq d-1$. Since $\operatorname{dim} S / I_{d-1}=d-1$, the assertion follows.

The proof of Corollary 1.5 provides the following additional information.

Corollary 1.6. We have $\operatorname{depth} S / I_{j-1} \leq \operatorname{depth} S / I_{j} \leq \operatorname{depth} S / I$ for all $0 \leq j \leq \operatorname{dim} S / I$.

\section{Applications to Stanley Decompositions}

Let $I \subset S$ be a monomial ideal. In the recent paper [4] it was shown that the Stanley depth of $S / I$ can be computed by means of properties of $P_{S / I}^{g}$. The result [4, Theorem 2.1] can be summarized as follows: given any poset $P$ and $a, b \in P$, we set $[a, b]=\{c \in$ $P: a \leq c \leq b\}$ and call $[a, b]$ an interval. Of course, $[a, b] \neq \emptyset$ if and only if $a \leq b$. Suppose $P$ is a finite poset. A partition of $P$ is a disjoint union

$$
\mathcal{P}: \quad P=\bigcup_{i=1}^{r}\left[c_{i}, d_{i}\right]
$$

of non-empty intervals. Let $\mathcal{P}: P_{S / I}^{g}=\bigcup_{i=1}^{r}\left[c_{i}, d_{i}\right]$ be a partition of $P_{S / I}^{g}$. We set

$$
\rho(\mathcal{P})=\min \left\{\rho\left(d_{i}\right): i=1, \ldots, r\right\} .
$$

Then

$$
\operatorname{sdepth} S / I=\max \left\{\rho(\mathcal{P}): \mathcal{P} \text { is a partition of } P_{S / I}^{g}\right\}
$$


We use this characterization of the Stanley depth and the results of the previous section to prove

Proposition 2.1. For all $0 \leq j \leq d=\operatorname{dim} S / I$ we have

$$
\text { sdepth } S / I \geq \operatorname{sdepth} S / I_{j} \text {. }
$$

Proof. Observe that $P_{S / I_{j}}^{g}=\left\{a \in P_{S / I}^{g}: \rho(a) \leq j\right\}$. Let $t$ be the Stanley depth of $S / I_{j}$. Then there exists a partition $\mathcal{P}: P_{S / I_{j}}^{g}=\bigcup_{i=1}^{r}\left[c_{i}, d_{i}\right]$ with $\rho(\mathcal{P})=t$. We complete the partition of $\mathcal{P}$ to a partition $\mathcal{P}^{\prime}$ of $S / I$ by adding the intervals $[a, a]$ with $a \in P_{S / I}^{g} \backslash P_{S / I_{j}}^{g}$. Since $\rho(a)>j$ for all $a \in P_{S / I}^{g} \backslash P_{S / I_{j}}^{g}$ it follows that $\rho\left(\mathcal{P}^{\prime}\right)=t$. Hence $\operatorname{sdepth} S / I \geq t$, as desired.

We call an algebra of the form $S / I$ a monomial factor algebra if $I \subset S$ is a monomial ideal. Stanley's conjecture for a monomial factor algebra $S / I$ says that $\operatorname{depth} S / I \leq$ sdepth $S / I$.

Corollary 2.2. Suppose Stanley's conjecture holds for all Cohen-Macaulay monomial factor algebras of dimension $t$. Then the conjecture holds for all monomial factor algebras of depth $t$. In particular, Stanley's conjecture holds for all monomial factor algebras if and only it holds for all Cohen-Macaulay monomial factor algebras.

Proof. Let $S / I$ be a monomial factor algebra with $t=\operatorname{depth} S / I$. Then $S / I_{t}$ is CohenMacaulay of dimension $t$, see Corollary 1.5. Our assumption implies that sdepth $S / I_{t}=t$. Thus the assertion follows from Proposition 2.1.

As a concrete application we have

Corollary 2.3. Let $S / I$ be a monomial factor algebra with depth $S / I \leq 1$. Then $S / I$ satisfies Stanley's conjecture.

Proof. According to Corollary 2.2 it suffices to show that any Cohen-Macaulay $K$-algebra $S / I$ of dimension $\leq 1$ satisfies Stanley's conjecture. This is trivially the case if $\operatorname{dim} S / I=0$, and has been shown if $\operatorname{dim} S / I=1$ in [1, Corollary 3].

We now prove a statement which in a certain sense is dual to that of Corollary 2.2. Let $I \subset J$ be monomial ideals and $\mathcal{D}=\bigoplus_{i=1}^{r} x^{c_{i}} K\left[Z_{i}\right]$ a Stanley decomposition of $J / I$. The number $\max \left\{\left|c_{i}\right|: i=1, \ldots, r\right\}$ is called the $h$-regularity of $\mathcal{D}$, denoted by $\operatorname{hreg}(\mathcal{D})$.

We set

$$
\operatorname{hreg}(J / I)=\min \{\operatorname{hreg}(\mathcal{D}): \mathcal{D} \text { is a Stanley decomposition of } J / I\}
$$

and call this number the $h$-regularity of $J / I$. In [7], the second author conjectured that $\operatorname{hreg}(J / I) \leq \operatorname{reg}(J / I)$. 
Let $g \in \mathbb{N}^{n}$ with $g \geq a$ for all minimal monomial generators $x^{a}$ of $I$ and $J$. For a partition $\mathcal{P}: P_{J / I}^{g}=\bigcup_{j=1}^{r}\left[c_{i}, d_{i}\right]$, we set

$$
\sigma(\mathcal{P})=\max \left\{\sigma_{i}(\mathcal{P}): i=1, \ldots, r\right\},
$$

where

$$
\sigma_{i}(\mathcal{P})=\max \left\{|c|: c \in\left[c_{i}, d_{i}\right] \text { and } c(j)=c_{i}(j) \text { for all } j \text { with } x_{j} \in Z_{d_{i}}\right\} .
$$

Proposition 2.4. $\operatorname{hreg}(J / I)=\min \left\{\sigma(\mathcal{P}): \mathcal{P}\right.$ is a partition of $\left.P_{J / I}^{g}\right\}$.

Proof. To each partition $\mathcal{P}: P_{J / I}^{g}=\bigcup_{j=1}^{r}\left[c_{i}, d_{i}\right]$ belongs a Stanley decomposition $\mathcal{D}(\mathcal{P})$, as described in [4, Theorem 2.1(a)]. The assignment is such that $\operatorname{hreg}(\mathcal{D}(\mathcal{P}))=\sigma(\mathcal{P})$. This shows that $\operatorname{hreg}(J / I) \leq \min \left\{\sigma(\mathcal{P}): \mathcal{P}\right.$ is a partition of $\left.P_{J / I}^{g}\right\}$.

In order to prove that equality holds, we need to find a partition $\mathcal{P}$ with $\operatorname{hreg}(J / I)=$ $\sigma(\mathcal{P})$. Let $\mathcal{D}: \quad J / I=\bigoplus_{i=1}^{r} x^{c_{i}} K\left[Z_{i}\right]$ be a Stanley decomposition of $J / I$ with $\operatorname{hreg}(\mathcal{D})=$ $\operatorname{hreg}(J / I)$. In [4, Theorem 2.1(b)] it is shown $\mathcal{P}: P_{J / I}^{g}=\bigcup_{i, c_{i} \leq g}\left[c_{i}, d_{i}\right]$ is a partition of $P_{J / I}^{g}$, where $d_{i}(j)=c_{i}(j)$ if $x_{j} \notin Z_{i}$, and $d_{i}(j)=g(j)$ otherwise. Thus we see that $\operatorname{hreg}(J / I)=\max \left\{\left|c_{i}\right|: i=1, \ldots, r\right\}=\sigma(\mathcal{P})$.

Observe that the preceding proposition implies in particular that $\mathrm{hreg}(J / I)$ can be computed in a finite number of steps.

For a graded ideal $I$ we denote by $I_{\geq j}$ the $j$ th truncation of $I$, that is, the ideal generated by all homogeneous elements $f \in I$ with $\operatorname{deg} f \geq j$.

Proposition 2.5. For all $j \geq 0$ we have $\operatorname{hreg}(I) \leq \operatorname{hreg}\left(I_{\geq j}\right)$.

Proof. We choose $g \in \mathbb{N}^{n}$ such that $g \geq a$ for all generators $x^{a}$ of $I$ and $I_{\geq j}$. Let $\mathcal{P}$ be a partition of $P_{I_{\geq j}}^{g}$ with $\sigma(\mathcal{P})=\operatorname{hreg}\left(I_{\geq j}\right)$. We complete the partition $\mathcal{P}$ to a partition $\mathcal{P}^{\prime}$ of $P_{I}^{g}$ by adding the intervals $[a, a]$ with $a \in P_{I}^{g} \backslash P_{I_{\geq j}}^{g}$. Then Proposition 2.4 implies that $\operatorname{hreg}(I) \leq \sigma\left(\mathcal{P}^{\prime}\right)=\sigma(\mathcal{P})=\operatorname{hreg}\left(I_{\geq j}\right)$.

Corollary 2.6. Suppose $\operatorname{hreg}(I) \leq \operatorname{reg}(I)$ for all ideals with linear resolution. Then this inequality is valid for all graded ideals.

Proof. By a result of Eisenbud and Goto [3] (see also [2, Theorem 4.3.1]) one has

$$
\operatorname{reg}(I)=\min \left\{j: I_{\geq j} \text { has a linear resolution }\right\} .
$$

We choose a $j$ such that $I_{\geq j}$ has a linear resolution. Then our assumption and Proposition 2.5 imply that $\operatorname{hreg}(I) \leq \operatorname{hreg}\left(I_{\geq j}\right) \leq \operatorname{reg}\left(I_{\geq j}\right)=\operatorname{reg}(I)$.

For a monomial ideal $I$ with linear resolution, minimally generated by $x^{a_{1}}, \ldots, x^{a_{r}}$, Soleyman-Jahan's conjecture reads as follows: there exist $b_{1}, \ldots, b_{r} \in P_{I}^{g}$ such that $P_{I}^{g}=\bigcup_{i=1}^{r}\left[a_{i}, b_{i}\right]$. In other words, $P_{I}^{g}$ can be partitioned by intervals whose lower ends correspond to the generators of $I$. 


\section{REFERENCES}

[1] J. Apel, On a conjecture of R. P. Stanley; Part II - Quotients Modulo Monomial Ideals, J. of Alg. Comb. 17, (2003), 57-74.

[2] W. Bruns, J. Herzog, Cohen Macaulay rings, Revised Edition, Cambridge, 1996.

[3] D. Eisenbud, S. Goto, Linear free resolutions and minimal multiplicity, J. Alg. 88 (1983), 202-224.

[4] J. Herzog, M. Vladoiu, Xinxian Zheng, How to compute the Stanley depth of a monomial ideal, to appear in J. Alg.

[5] T. Hibi, Quotient Algebras of Stanley-Reisner Rings and Local Cohomology, J. Alg. bf 140, (1991), 336-343.

[6] D. Smith, On the Cohen-Macaulay property in commutative algebra and simplicial topology, Pacific J. Math. 141, (1990), 165-196.

[7] A. Soleyman-Jahan, Stanley decompositions of squarefree modules and Alexander duality, arXiv:math.AC/0709.4145, preprint 2007.

[8] R. P. Stanley, Linear Diophantine equations and local cohomology, Invent. Math. 68, (1982), 175-193.

Jürgen Herzog, Fachbereich Mathematik und Informatik, Universität Duisburg-Essen, Campus Essen, 45117 Essen, Germany

E-mail address: juergen.herzog@uni-due.de

Ali Soleyman Jahan, Fachbereich Mathematik und Informatik, Universität DuisburgEssen, Campus Essen, 45117 Essen, Germany

E-mail address: ali.soleyman-jahan@stud.uni-due.de

Xinxian Zheng, Fachbereich Mathematik und Informatik, Universität Duisburg-Essen, CamPus Essen, 45117 Essen, Germany

E-mail address: xinxian.zheng@uni-due.de 